\title{
Comparative Study of TiN/SiC and CrN/SiC multilayer systems produced by PVD.
}

\author{
J.L. Bernal ${ }^{1}$, E. Vera $^{2}$, A.I. Martínez ${ }^{3}$, A. García ${ }^{4}$ \\ 1. Mechanics Department, TecNM / Instituto Tecnológico de Orizaba, Orizaba, México. \\ 2. Mechanics Department, TecNM / Instituto Tecnológico de Pachuca, Pachuca, México. \\ 3. Automotive Mechanics Department, Universidad Politécnica de Pachuca, Pachuca, México. \\ 4. Faculty of Science, Universidad Autónoma de San Luis Potosí, San Luis Potosí, México.
}

Nowadays, the development of advanced materials with mechanical, tribological and anticorrosive properties has attracted great interest from technologists and researchers [1-2]. In particular with regard to the development of multilayer materials, the aim is to solve the problem of formation of micro-cracks originated during the wear condition, which causes the fracture in the material. A proposed solution consists of developing layers on the substrate with certain affinity in fracture toughness, as well as the creation of a dislocation interface that prevents the propagation of the corrosive medium to the substrate and the propagation of micro-cracks [3]. In the present investigation, two coating configurations by PVD are micro-structurally compared $\mathrm{TiN} / \mathrm{SiC}$ system deposited on a substrate of low alloy steel AISI 4140 [4], and $\mathrm{CrN} / \mathrm{SiC}$ system deposited by the same technique on the same type of substrate [5].

The TiN and CrN coatings were produced in a BAI 1200 machine at Oerlikon Balzers in México. They were deposited on 4140 alloy steel substrate with a high vacuum and a temperature of $450^{\circ} \mathrm{C}$ for 2 hours. Then, $\mathrm{SiC}$ was deposited under the same conditions on $\mathrm{CrN}$ and $\mathrm{TiN}$ layers introducing the precursor of hexamethyldisilizane (HMDS) $\mathrm{CH} 3 \mathrm{SiNHSiCH} 3$ and argón as a process gas inside the plasma reactor at variable pressures of the order of 0.8 atm. In both cases, the ionization potentially fluctuated from $700 \mathrm{~V}$ to $900 \mathrm{~V}$, and the substrate temperatures were around $600{ }^{\circ} \mathrm{C}$. The film growth rate on average is approx. $1 \mu \mathrm{m} / \mathrm{h}$ in both systems and its average hardness in each case is approx. $\sim 9$ on the Mohs scale.

Figures 1 and 2 respectively show Scanning Electron Microscopic (SEM) images of the TiN/SiC and $\mathrm{CrN} / \mathrm{SiC}$ systems that confirm the presence of bilayers on an AISI 4140 substrate. Figures 3 and 4 respectively show their Secondary electrons images (SEI) of the previously mentioned systems. The XRD scans (Figure 5) confirm the presence of a single alpha phase of SiC that precipitate at (103), (105) and (109) orientations on TiN layer. Nevertheless, the presence of an alpha phase of SiC is not observed in the X-Ray Diffraction Pattern of $\mathrm{CrN} / \mathrm{SiC}$ system, which is possibly due to the non-existence of a crystalline phase or little crystallinity of the $\mathrm{SiC}$ layer.

\section{References:}

[1] E. Marin et al, Materials and Design, 89 (2016), p.314-322.

[2] S.A Naghibi, K. Raeissi, M.H. Fahti. Materials Chemistry and Physics, 148 (2014), p. 614-623.

[3] Flores M, Muhl S, Huerta L. Surf. Coat. Technol. 200 (2005) 1315-1319.

[4] J. L. Bernal et al. Microsc. Microanal. 22 (Suppl 3) 2016, p. 1984,1985.

[5] J. L. Bernal et al. Microsc. Microanal. 23 (Suppl 1) 2017, p. 416, 417. 


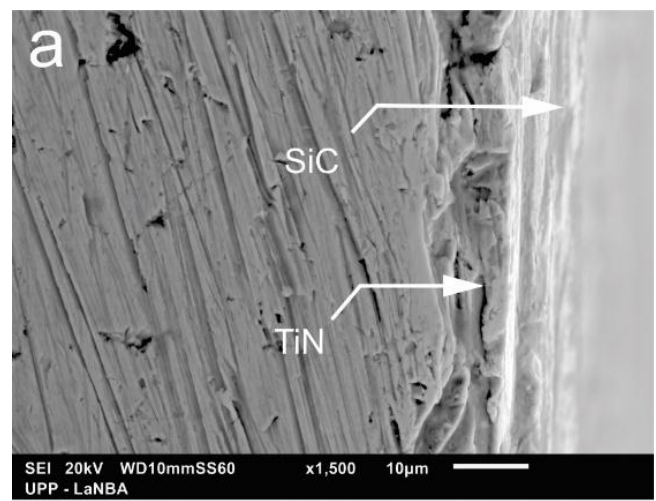

Figure 1. SEM cross-sectional micrograph of $\mathrm{TiN} / \mathrm{SiC}$.

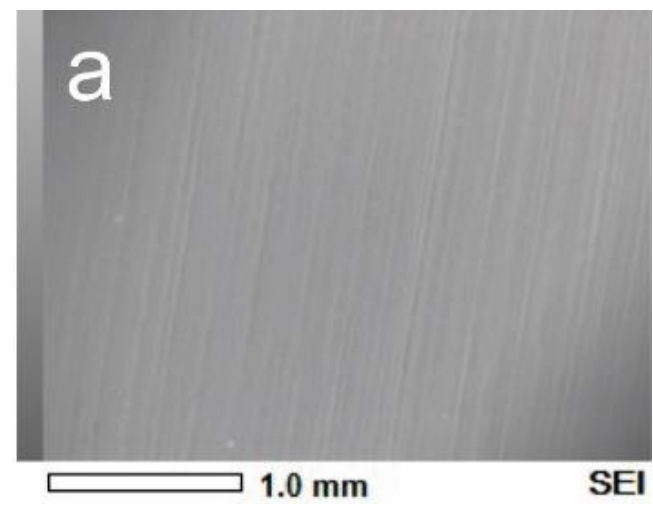

Figure 3. Secondary Electron Image (SEI) of the SiC surface corresponding to the TiN/SiC system.

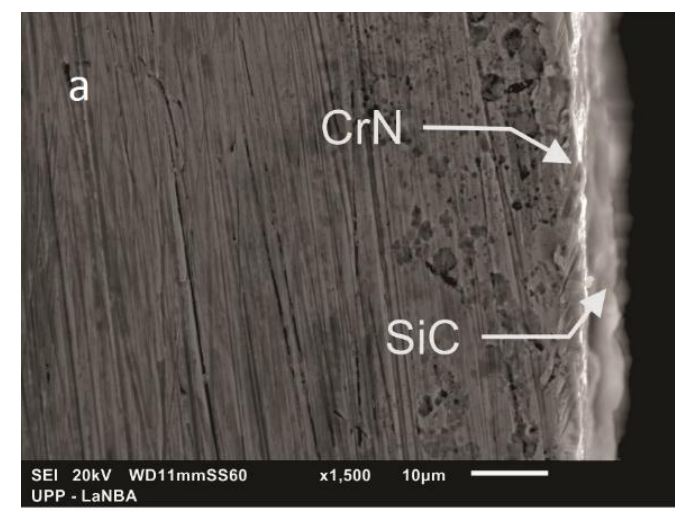

Fig. 2. SEM cross-sectional micrograph of $\mathrm{CrN} / \mathrm{SiC}$.

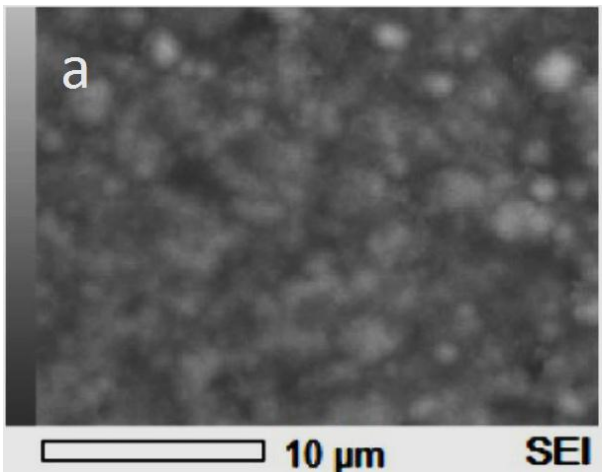

Figure 4. Secondary Electron Image (SEI) of the $\mathrm{SiC}$ surface corresponding to the $\mathrm{CrN} / \mathrm{SiC}$ system.

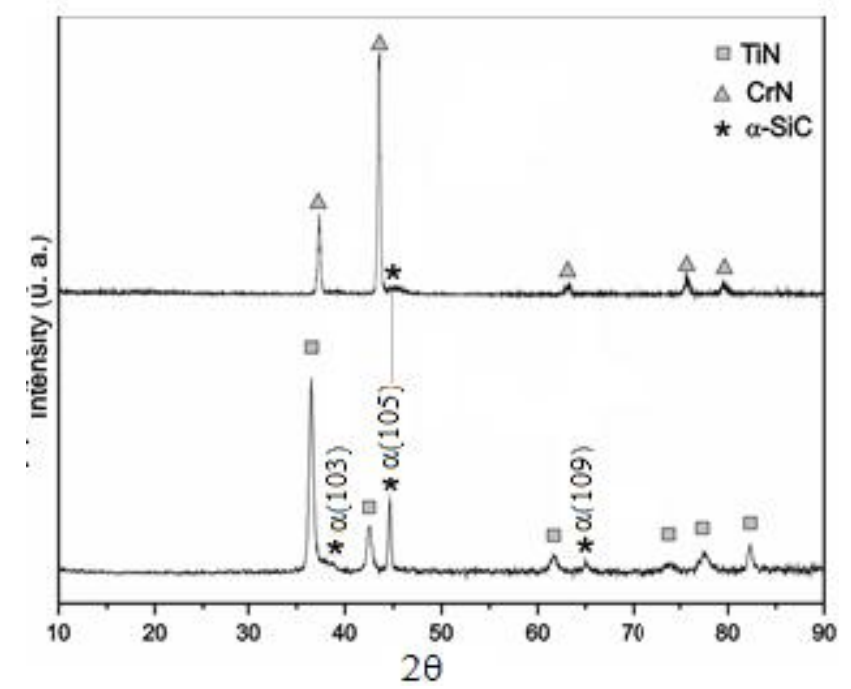

Fig. 5. XRD diffraction patterns of $\mathrm{TiN}, \mathrm{CrN}$ and $\mathrm{SiC}$. 